EOMmun: Communication et organisation

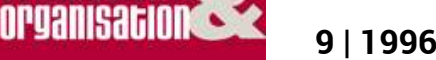

La communication des institutions religieuses

\title{
Entretien avec Jean-Michel Di Falco
}

\section{Hugues Hotier}

\section{OpenEdition}

Journals

Édition électronique

URL : http://journals.openedition.org/communicationorganisation/1855

DOI : 10.4000/communicationorganisation. 1855

ISSN : 1775-3546

\section{Éditeur}

Presses universitaires de Bordeaux

\section{Édition imprimée}

Date de publication : 1 mai 1996

ISSN : 1168-5549

\section{Référence électronique}

Hugues Hotier, «Entretien avec Jean-Michel Di Falco », Communication et organisation [En ligne], 9 | 1996, mis en ligne le 26 mars 2012, consulté le 01 mai 2019. URL : http://journals.openedition.org/ communicationorganisation/1855 ; DOI : 10.4000/communicationorganisation.1855

Ce document a été généré automatiquement le 1 mai 2019

(c) Presses universitaires de Bordeaux 


\title{
Entretien avec Jean-Michel Di Falco
}

\author{
Hugues Hotier
}

\section{NOTE DE L'ÉDITEUR}

Entretien réalisé par Hugues Hotier

1 HH: Vous avez été le porte-parole de la Conférence des Évêques de France jusqu'au 1er janvier dernier. La dénomination est précise, mais cependant souvent modifiée par ceux qui l'emploient. Et l'on entend parler de vous comme du "porte-parole de l'Église (de France)». Réfutez-vous cette appellation? Quelles différences faites-vous entre les deux?

2 J-M.DF : Je réfute sans hésitation cette appellation de "porte-parole de l'Église de France ». Cette confusion, plus que fréquente, n'est pas pour autant une simple erreur sémantique. Elle est révélatrice de difficultés particulièrement intéressantes à analyser. Le concept de «porte-parole de l'Église de France» est faux sur le terrain de l'ecclésiologie, c'est-à-dire qu'il n'a pas de sens par rapport à ce qu'est réellement l'Église. Mais si tant de gens font cette confusion, c'est précisément parce que l'Église a de plus en plus de mal à faire comprendre ce qu'elle est, tant en interne qu'en externe !

D'abord, il n'y a pas d'église «de France », mais c'est seulement une partie de l'Église universelle qui se trouve "en France ». Il est difficile de faire comprendre en quelques mots cette première distinction, pourtant essentielle, car si les chrétiens de France forment bien là où ils sont des communautés au sens plein du terme, celles-ci n'ont de raison d'être, de substance dirons-nous, que parce qu'elles sont en communion dans un même corps, unique, celui de l'Église universelle.

Ensuite, il ne peut y avoir de "porte-parole» de cette église en France, car chaque chrétien est légitiment porte-parole de l'Église dont il est membre à part entière. Seuls des groupes de chrétiens peuvent se donner un porte-parole, avec le plein accord de chacun. C'est ce que font les évêques. La difficulté à comprendre cette seconde distinction vient de ce que l'on confond souvent l'église, ensemble de tous les baptisés, avec l'Église - 
hiérarchie. Mais il s'agit à nouveau d'une confusion grave car que reste-t-il d'un corps humain si on le réduit à sa tête?

6 Voilà un rapide aperçu des erreurs que renferme l'expression de « porte-parole de l'Église de France ", mais il faudrait tout un numéro de votre revue pour en ébaucher les causes et les conséquences !

$7 \mathrm{HH}$ : Le fait que la Conférence des Évêques de France ait un porte-parole signifie-t-elle que l'Église se plie aux fonctionnements de la société dans laquelle elle est, ou bien peut-on l'interpréter comme une difficulté plus grande que naguère à faire entendre sa voix, sa parole?

8 J-M. DF : Il est dans la logique d'une "Conférence des Évêques » de se doter d'un porteparole. La structure des conférences épiscopales est toute neuve à l'aune de l'histoie de l'Église: elle date du Concile Vatican II. Elle ne remplace pas le fonctionnement traditionnel de l'Église Universelle, selon lequel chaque évêque détient son autorité directement du pape, une autorité à la fois individuelle et propre sur le territoire de son diocèse, et à la fois collégiale au plan universel, dans la conversation de la foi. Un évêque n'est donc ni un préfet, ni un député !

9 Et pourtant, l'Église a, plus que le souci, le devoir de s'adapter au fonctionnement de la société dans laquelle elle vit. Elle l'a fait de tout temps. Il faudrait faire un peu d'histoire pour constater à quel point les structures ecclésiales et civiles s'influencent mutuellement depuis des siècles. La création des Conférences épiscopales provient du constat que sur de nombreux sujets, il est nécessaire aux évêques d'un même pays, et d'un même continent comme c'est déjà le cas en Europe et en Amérique latine, d'avoir une structure au sein de laquelle ils peuvent se concerter, réfléchir ensemble et s'exprimer d'une seule voix. Étant donné les règles du jeu médiatique, cette « voix », si elle veut être entendue, doit être portée par un individu désigné es qualité : c'est le rôle du porteparole. Depuis 1962, huit personnes se sont succédées à ce poste.

10 Mais il faut bien comprendre que, tout comme la conférence épiscopale n'annule pas l'autorité individuelle de chaque évêque, l'existence d'un porte-parole ne leur retire pas leur liberté individuelle de s'exprimer.

$11 \mathrm{HH}$ : Si je me réfere à la presse, la Conférence des Évêques de France envisage d'élaborer un plan de communication. Peut-on dire qu'on passe du stade de l'information symbolisée par l'expression "porte-parole », à celui de la communication stratégique?

12 J-M. DF : Il est difficile de répondre à la question en ces termes. D'un côté en effet, l'Église n'a pas d'autre « communication stratégique » que celle que lui prescrit l'Évangile : aller, parmi les nations, proclamer la mort et la résurrection de Jésus Christ. Cela dure depuis 2000 ans, et avouez que cela n'a pas trop mal réussi : de 12 convaincus dans un petit pays du bassin méditerranéen, on compte aujourd'hui plus de 1,5 milliard de chrétiens sur les cinq continents. Pour peu que l'on veuille bien quitter un instant nos œillères européennes, il faut bien avouer que le mouvement continue : 11000 adultes baptisés en Corée du Sud pour la seule année 1996,.... Mais cette communication stratégique-là n'est pas l'affaire d'un seul porte-parole, ni même d'un groupe défini de missionnaires ou autre: c'est la raison d'être de l'Église tout entière. Depuis 2000 ans, l'Église n'est que communication!

13 Mais d'un autre côté, je serais malhonnête de m'en tenir là dans ma réponse : il est évident qu'après une quinzaine d'années passées dans le domaine de la communication de l'Église, au sens plus classique du rapport Eglise-média, je suis mieux placé que beaucoup pour reconnaître toutes nos faiblesses : manque de moyen, de savoir-faire, ou 
parfois seulement de courage et de volonté ! Aujourd'hui, la question devient plus pressante pour de nombreuses raisons: on peut citer l'accroissement relatif de l'éloignement de la société française par rapport à l'Église, mais il ne faut pas négliger non plus les motifs plus internes, comme l'arrivée prochaine d'une période de rupture importante qui va nous obliger à revoir tout notre mode de fonctionnement, lorsque les générations encore nombreuses de prêtres vont s'effacer et que va se faire sentir avec force la crise des vocations.

est-il sans doute nécessaire aujourd'hui de mettre un certain nombre de choses à plat, et de repenser de façon plus rationnelle peut-être les structures que l'Église se donne pour être en relation avec les médias.

HH: Quels ont été les moments les plus difficiles dans l'exercice de votre fonction au cours de ces huit années, les paroles les plus difficiles à transmettre? Quels ont été les obstacles les plus difficiles à surmonter, les réceptions les plus difficiles à obtenir? $\mathrm{J}-\mathrm{M}$. DF : Vaste problème, tant les huit dernières années ont été riches en actualités diverses concernant, ou plutôt « impliquant » l'Église! Une simple énumération de faits difficiles à gérer n'aurait pas, je crois, beaucoup de sens.

Si je devais tenter une synthèse, j'écarterai d'abord, même si cela peut sembler une hérésie pour un communicateur orthodoxe, les problèmes de popularité : l'Église ne cherche pas comme objectif premier à développer un message qui plaise. Son but principal est bien plus d'être dans la vérité par rapport au message qu'elle détient du Christ lui-même, et ensuite de faire comprendre ce message aux hommes de son temps. Cette " mise en forme ", qui n'est pas, encore une fois, une " mise au goût du jour ", passe aujourd'hui par le canal des médias, et particulièrement par celui de l'audiovisuel.

S'il fallait en un mot résumer le point sur lequel j'ai concentré mes efforts comme porteparole des Évêques de France, je dirais que ma philosophie a un peu été celle de Saint Exupéry : il faut s'apprivoiser, il faut que l'église et les médias accèdent enfin à un rapport de confiance réciproque, et que nous sortions du rapport biaisé de fascination-répulsion dans lequel nous sommes bien souvent prisonnier. Cela passe par une meilleure connaissance réciproque. Il faut admettre qu'aujourd'hui l'Église est bien seule à se soucier de la façon dont elle va pouvoir faire un bout de chemin vers les médias. En face, rare sont les rédactions, pour prendre un seul exemple, où l'on se soucient compétent... C'est grave, car je suis convaincu que l'on ne peut pas comprendre le monde dans lequel nous vivons sans prendre en compte le facteur religieux, et pour cela, deux ou trois clichés, ou quelques souvenirs de catéchisme, ne suffisent pas!

Allons plus loin: la principale difficulté pour l'Église aujourd'hui est de savoir jusqu'à quel point son message est compatible avec les règles de la communication moderne. Doit-on sans sourciller entrer dans un système où le vraisemblable a plus de valeur que le vrai ? Où tout doit être dit en 35 secondes? Où l'émotion est le principal ressort de communication? Si les outils de communication étendent aujourd'hui leurs réseaux comme jamais sans doute dans l'histoire de l'homme, il faut admettre aussi que les règles du jeu sont de plus en plus uniformes, de plus en plus gouvernées par les lois du commerce et de la concurrence, or l'Église n'a rien à vendre.

21 HH: Dans l'accomplissement de votre mission, vous avez été aidé sans doute par votre foi, par le fait que vous adhériez aux propos que vous aviez à tenir, par votre intelligence aussi. Cela dit, vous disposez d'un autre atout de nature physique: un timbre de voix radiophonique, une apparence 
télégénique... quelle part accordez-vous à ces qualités physiques personnelles dans le succès de votre mission?

J-M. DF : Pour être tout à fait franc, j'accorde à ce facteur une part très ambigüe ! D'une part, il m'a évidemment servi à «faire mon trou ", à emmerger dans un monde d'apparence où règne le star system. Je serai naïf de nier ce facteur, comme il serait à mon avis naïf de nier l'impact de la capeline et de la barbe blanche d'un abbé Pierre ou de la soutane blanche d'un pape, même si ni l'un ni l'autre n'ont évidemment, choisit leur apparence pour cela. Il faut reconnaître un certain "génie médiatique » aux habits ecclésisiastiques : imagine-t-on un pape en costume et cravate? A l'inverse, les religieuses chères au cœur des publicitaires portent encore la cornette que l'on ne trouve plus dans les couvents depuis 30 ans!

Mais dans le même temps, je vous avoue que cet aspect de ma personalité a nourri bien des procès et des innimitiés. Vous me permettrez de ne pas m'apesantir sur le sujet... $\mathrm{HH}$ : Quelle est exactement la nouvelle mission qui vous a été confiée? J-M. DF : Je quitte pour un temps le contact direct avec le monde des médias. Comme attaché culturel de l'ambassade de France près le Saint Siège et directeur du centre culturel Saint Louis de France, je suis désormais chargé des relations culturelles entre notre pays et le Vatican: c'est un domaine vaste, et qui possède de nombreuses passerelles avec le monde de la communication : les médias ne sont-ils pas aujourd'hui les premiers artisans de la culture contemporaine? 